\title{
Tactile Slip and Hand Displacement: Bending Hand Motion with Tactile Illusions
}

\author{
Matteo Bianchi ${ }^{1^{* \dagger}}$, Alessandro Moscatelli ${ }^{2 * \dagger}$, \\ Simone Ciotti ${ }^{1,3}$, Gemma Carolina Bettelani ${ }^{1}$, Federica Fioretti ${ }^{1}$, \\ Francesco Lacquaniti ${ }^{2}$ and Antonio Bicchi ${ }^{1,3}$
}

\begin{abstract}
Touch provides an important cue to perceive the physical properties of the external objects. Recent studies showed that tactile sensation also contributes to our sense of hand position and displacement in perceptual tasks. In this study, we tested the hypothesis that, sliding our hand over a stationary surface, tactile motion may provide a feedback for guiding hand trajectory. We asked participants to touch a plate having parallel ridges at different orientations and to perform a self-paced, straight movement of the hand. In our daily-life experience, tactile slip motion is equal and opposite to hand motion. Here, we used a well-established perceptual illusion to dissociate, in a controlled manner, the two motion estimates. According to previous studies, this stimulus produces a bias in the perceived direction of tactile motion, predicted by tactile flow model. We showed a systematic deviation in the movement of the hand towards a direction opposite to the one predicted by tactile flow, supporting the hypothesis that touch contributes to motor control of the hand. We suggested a model where the perceived hand motion is equal to a weighted sum of the estimate from classical proprioceptive cues (e.g., from musculoskeletal system) and the estimate from tactile slip.
\end{abstract}

\section{INTRODUCTION}

Cutaneous touch plays an important role in the perception of the physical properties (e.g., shape, texture, weight) and the motion status of external objects [1], [2]. Material properties of the object, such as roughness [1], [3] and compliance [4], [5], are also encoded by the tactile system. In addition to this role in object and material perception, the deformation of the fingertip also contributes to our sense of hand position and motion [6], [7]. Classical studies in physiology showed that receptors in the musculoskeletal system (such as muscle spindle, Golgi tendon organ and joint receptors) and strain patterns on the skin convey information on the static position and the displacement of our limbs [8], [9], [10], [11], [12], [13]. Specific cutaneous stimuli can also produce the illusory sensation of hand motion in perceptual tasks. We recently showed that the deformation of the fingertip's skin occurring when we push the finger against a soft surface generates

\footnotetext{
${ }^{1}$ M. Bianchi, S. Ciotti, G. C. Bettelani, F. Fioretti A. Bicchi are with Centro di Ricerca "E. Piaggio", Università di Pisa, Pisa, Italy and with the Department of Information Engineering of Università di Pisa, Pisa, Italy. \{m.bianchi\} at centropiaggio.unipi.it

2 A. Moscatelli and F. Lacquaniti are with Department of Systems Medicine and Centre of Space Bio-medicine, University of Rome "Tor Vergata", Rome, Italy and with the Laboratory of Neuromotor Physiology, IRCCS Santa Lucia Foundation, Rome, Italy. $\{a . m o s c a t e l l i\}$ at hstantalucia.it

3 A. Bicchi and S. Ciotti are also with the Department of Advanced Robotics, Istituto Italiano di Tecnologia, Genova, Italy.

* These authors contributed equally to this work.

$\dagger$ Corresponding authors.
}

the illusory sensation of finger displacement [6]. The tactile motion generated by a rotating disk produces the sensation of the hand rotating in the opposite direction of the disk [14].

In this study, we tested the hypothesis that touch provides auxiliary cues to guide hand displacement [15], [16]. In our daily-life experience, whenever we slide the hand over a stationary surface, like a working desk or a table, the velocity of tactile motion is equal and opposite to the hand velocity (Fig. 1). If the sensorimotor system uses touch as a motion cue, a stimulus that decouples the tactile and the kinesthetic velocity estimates would provide a biased motion signal and produce a systematic error in hand movement. Here, we used a well-established tactile phenomenon (previously investigated by some of the coauthors of the present study), to decouple the two velocity estimates. In [17], participants kept the hand world-stationary and a textured plate with parallel raised ridges slid under their fingertip. The perceived movement of the plate was strongly biased towards a direction perpendicular to the orientation of the ridges, in accordance with the tactile flow model [17].

\section{Tactile Motion}

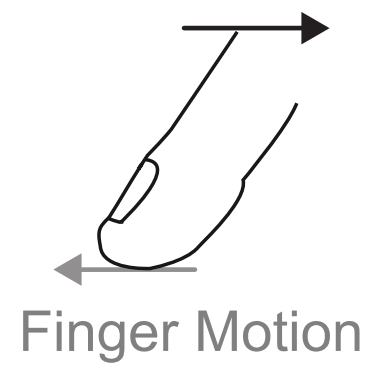

Fig. 1: Sliding the hand over a stationary surface, for example a working desk, the relative movement sensed from cutaneous touch (black) is equal and opposite to hand motion (grey). Therefore, touch can be a strong cue to hand motion.

Here, we used this perceptual phenomenon to decouple the tactile and proprioceptive feedback to active hand motion. We asked blindfolded participants to slide the finger on a surface with parallel ridges, trying to move the hand along a straight direction. According to our hypothesis, in the absence of other sensory feedback, tactile feedback should lead to a systematic error in hand displacement, towards a direction opposite to the one predicted by the tactile flow. Finally, we suggested a model (to be further evaluated in future studies) where the perceived hand motion is equal to a weighted sum of the estimate from classical proprioceptive cues (e.g., from receptors in the musculoskeletal 
system) and the estimate from tactile slip. Previous studies in psychophysics supported the hypothesis of an integration of multiple cues for the perception of hand displacement (see for example [14], [7], [6]), however, this was never evaluated for the on-line control of the hand and the limb movement.

\section{Methods}

\section{A. Participants}

Six naive healthy participants took part in the experiment (4 males and 2 female, age: $25.1 \pm 1.2867$, mean \pm standard deviation). Participants were all right-handed. Participants reported no medical condition that could have affected the experimental outcomes.The testing procedures were approved by the Ethical Committee of the University of Pisa, in accordance with the guidelines of the Declaration of Helsinki for research involving human subjects. Informed consent was obtained from all participants involved in the study.

\section{B. Stimulus and Procedure}

The experimental setup (Fig. 2) included a 3D-printed circular plate (diameter: $15 \mathrm{~cm}$ ) placed over a load cell (Micro Load Cell, 0 to $780 \mathrm{~g}$, CZL616C from Phidgets, Calgary, AB-Canada). The plate had a textured surface with regularly spaced ridges (ridge height and width: $1 \mathrm{~mm}$; space between ridges: $10 \mathrm{~mm}$ ), consistently with [17]. A Leap Motion device (Leap Motion Inc., San Francisco, U.S.) was placed above the plate for hand tracking. We centered the reference system of the Leap Motion device in the center of the circular plate. The sampling frequency of the Leap Motion device was equal to $40 \mathrm{~Hz}$, which allows to correctly track hand motion at typical scanning speeds.

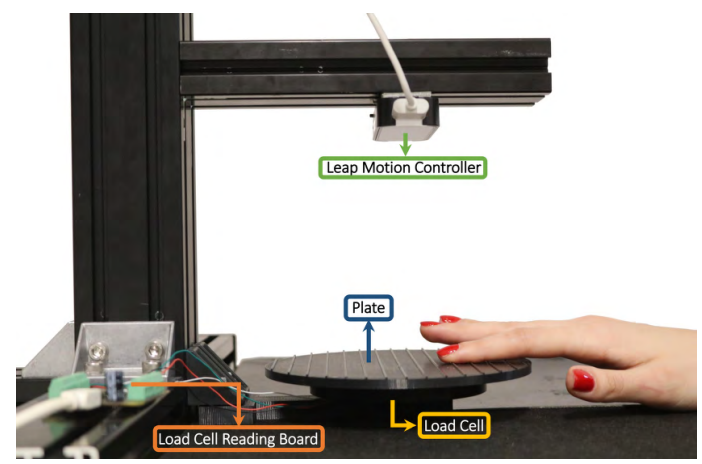

Fig. 2: The experimental setup including the textured circular plate, the Phidgets Micro Load Cell and the Leap Motion device.

Blindfolded participants sat on an office chair in front of the setup, with the center of the plate roughly aligned with their body mid-line. Headphones playing pink noise masked occasional ambient sound. In each trial, participants were required to contact the plate with their right index finger and to move the hand away from them along a straight path for approx. $10 \mathrm{~cm}$ (solid arrow in Fig. 3). Participants were instructed to contact the plate with a light touch.

Prior to each trial, the plate was rotated by the experimenter to one of the following angular position: $-60,-30$, $0,30,60$ deg. A zero angle means that the ridges of the plate were parallel to the frontal plane of the participant,

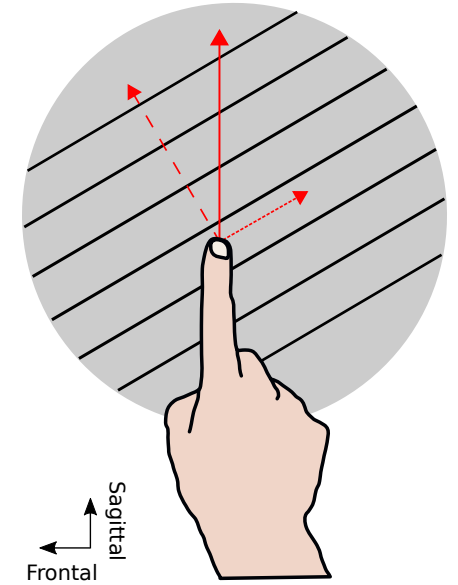

Fig. 3: Participants moved the hand on a plate with oblique ridges, along the direction indicated by the solid arrow. According to the model of tactile flow, the cutaneous feedback produced an illusory sensation of bending towards a direction perpendicular to the ridges (dashed arrow). This eventually led to an adjustment of the motion trajectory towards a direction orthogonal to tactile flow, i.e., parallel to the ridges (dotted arrow). The actual hand trajectory also depended on extra-cutaneous cues, e.g. from musculoskeletal system (not shown in the picture).

whereas negative (positive) angles means that the ridges were rotated clockwise (counterclockwise). Each stimulus orientation was presented fifteen times, in pseudo-random order. Additionally, participants replicated the task with a smooth plate without ridges. This aimed at correcting our results for possible biases in perceived direction introduced by extra-cutaneous cues, for e.g. proprioception [18]. Participants received no feedback about their performance during the experiment. At the end of each trial, the experimenter lifted the hand of the participant to place it back to the starting position.

Before the experimental session, participants underwent a training phase, where the experimenter instructed them to produce the right amount of force and hand displacement. During training, participants received a feedback whenever the actual force exceeded the threshold value of $2 \mathrm{~N}$.

\section{Data Analysis}

The hand trajectory was recorded with the tracking system of the apparatus and saved for the analysis. We linearly interpolated the hand trajectory separately for each trial and participant and estimated the angular deviation from a straight-ahead motion direction (i.e., the deviation from the solid arrow in Fig. 3). Negative (positive) angles means that the motion path rotated clockwise (counterclockwise) with respect to the solid arrow in the figure. Using Linear Mixed Model (LMM), we evaluated whether the orientation of the ridges, $\mathbf{X}$, predicted this angular error, $\mathbf{A}$ :

$$
\mathbf{A}=\beta_{0}+u_{0}+\left(\beta_{1}+u_{1}\right) \mathbf{X}+\varepsilon,
$$

where $\beta_{0}$ and $\beta_{1}$ are the fixed-effect intercept and slope, respectively, $u_{0}$ and $u_{1}$ are the random-effect intercept and slope of the model (between-participant variability), and $\varepsilon$ is the residual error term. In order to account for possible biases produced by extra-cutaneous cues (e.g., proprioceptive cues from the musculoskeletal system), we analyzed the trials with a zero-degree orientation of the ridged plate and with the 
smooth plate. First, we verified, using the Likelihood Ratio Test, that the angular error was not significantly different between these two experimental conditions. Then, we fitted the following model to estimate the angular deviation from straight direction in the absence of biasing tactile stimuli.

$$
\mathbf{A}_{\mathbf{0}}=\beta_{0}^{*}+u_{0}+\varepsilon,
$$

where $\mathbf{A}_{\mathbf{0}}$ is the predicted angle with zero-oriented or no ridges, and $\beta_{0}^{*}$ is the estimate of the possible bias due to extra-cutaneous cues. We used $\beta_{0}^{*}$ to correct the estimate of the tactile bias estimated in model (1). Next, we analyzed by means of LMM whether the orientation of the ridges predicted the final position error along the frontal plane, $\mathbf{P}$.

$$
\mathbf{P}=\eta_{0}+u_{0}+\left(\eta_{1}+u_{1}\right) \mathbf{X}+\varepsilon,
$$

In Equation (3), zero error means that the participant successfully moved straight, whereas positive (negative) error means that final position was to the left (right) of the body mid-line.

Additionally, we analyzed the force data to verify that participants were close to the required force threshold of 2 N. Normal forces were filtered with a second order, Butterworth low-pass filter (cutoff frequency equal to $10 \mathrm{~Hz}$ ). We saved the force peak from the filtered signal for each trial and participant that we retained for further analyses. Using LMM we evaluated whether the orientation of the ridges significantly affected the force peak, F. We used a second order polynomial to fit the data of the form:

$$
\mathbf{F}=\theta_{0}+u_{0}+\theta_{1} \mathbf{X}++\theta_{2} \mathbf{X}^{2}
$$

Data analysis was performed in $\mathrm{R}$ ( $\mathrm{R}$ version 3.3.2). Package lme4 was used to fit LMM [19]. In LMM (1) and (3), we tested the significance of the slope parameter, respectively $\beta_{1}$ and $\eta_{1}$, by means of the Likelihood Ratio Test.

\section{RESULTS}

Fig. 4 shows, for each participant (P01-P06), the angular deviation of the hand trajectory as a function of the orientation of the grating. Hand trajectory deviated towards a direction parallel to the ridges (effect size: $-0.15 \pm 0.03$, $\beta_{1} \pm$ Std. Error), in accordance with our hypothesis. That is, a clockwise rotation of the ridges with respect the frontal plane caused the participants to deviate hand motion from straight by bending leftwards, and vice versa. The effect was statistically significant $\left(\chi_{1}=7.0, \mathrm{p}<0.001\right)$. As shown in Figure 4, there is a significant offset in the linear function with larger absolute bias with clockwise rotated stimuli. We used model (2) to quantify the extra-cutaneous bias, independent of ridge orientation. This was equal to $4.2 \pm 1.925 \mathrm{deg}\left(\beta_{0}^{*} \pm\right.$ Std. Error). Correcting for the extracutaneous bias, the offset in model (1) was non-significantly different from zero, that is, the absolute bias was symmetric between clockwise- and counterclockwise-rotated stimuli.

The analysis of the position error confirmed this result. The final position shifted from left to right along the frontal plane depending upon the rotation (counterclockwise or clockwise) of the plate $\left(\eta_{1}=-0.03\right)$. The effect was statistically significant $\left(\chi_{1}=8.3, \mathrm{p}=0.004\right)$. Result from a representative participant are shown in Fig. 5, top panel.

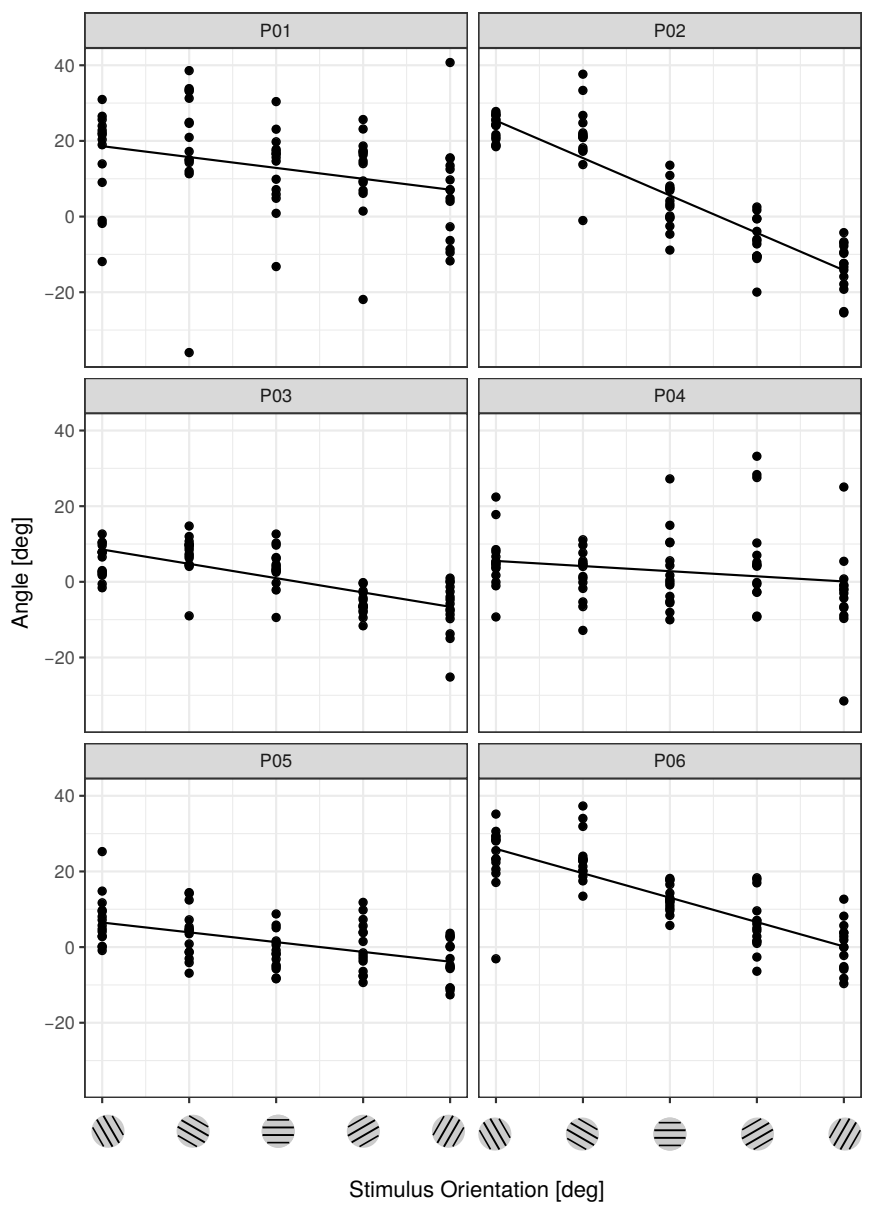

Fig. 4: The angular deviation of the hand trajectory as a function of the orientation of the grating in participants P01-P06. Point data for individual trial and LMM prediction.

It is worth noting that participants were not following the ridges. If this were the case, the absolute error would have been larger for $\pm 30 \mathrm{deg}$ stimuli and smaller for $\pm 60 \mathrm{deg}$, which was the opposite of what we found. This result is highlighted in Fig. 5. We asked to one participant to repeat the trial, in the same experimental conditions, but in this case he was explicitly required to follow the ridges (bottom panel in the figure). Following the ridges, we observed an angular deviation of $\pm 61 / 53 \mathrm{deg}$ for $\mp 30 \mathrm{deg}$, respectively, and $\pm 35 \mathrm{deg}$ for $\mp 60 \mathrm{deg}$, respectively. This trend is different from what we found in our experiments, where participants are instructed to move straight (top panel).

Average force was approximately equal to $2 \mathrm{~N}$ in participant P01 and smaller than $1 \mathrm{~N}$ on participants P02-P06. Grating orientation had little effect on force peak (Fig. 6). Force peak was maximum for the grating orientation parallel to the frontal plane. From LMM (4) we estimated the average value of force peak for a perpendicular (zero) orientation of the stimulus and this was equal to $0.92 \pm 0.23 \mathrm{~N}\left(\theta_{0} \pm\right.$ $\mathrm{SE}$ ). The difference in force peak between clockwise and counterclockwise ridges was small and equal to $0.04 \mathrm{~N}$ (peak at $60 \mathrm{deg}$ counterclockwise; minus peak at $60 \mathrm{deg}$ clockwise) 

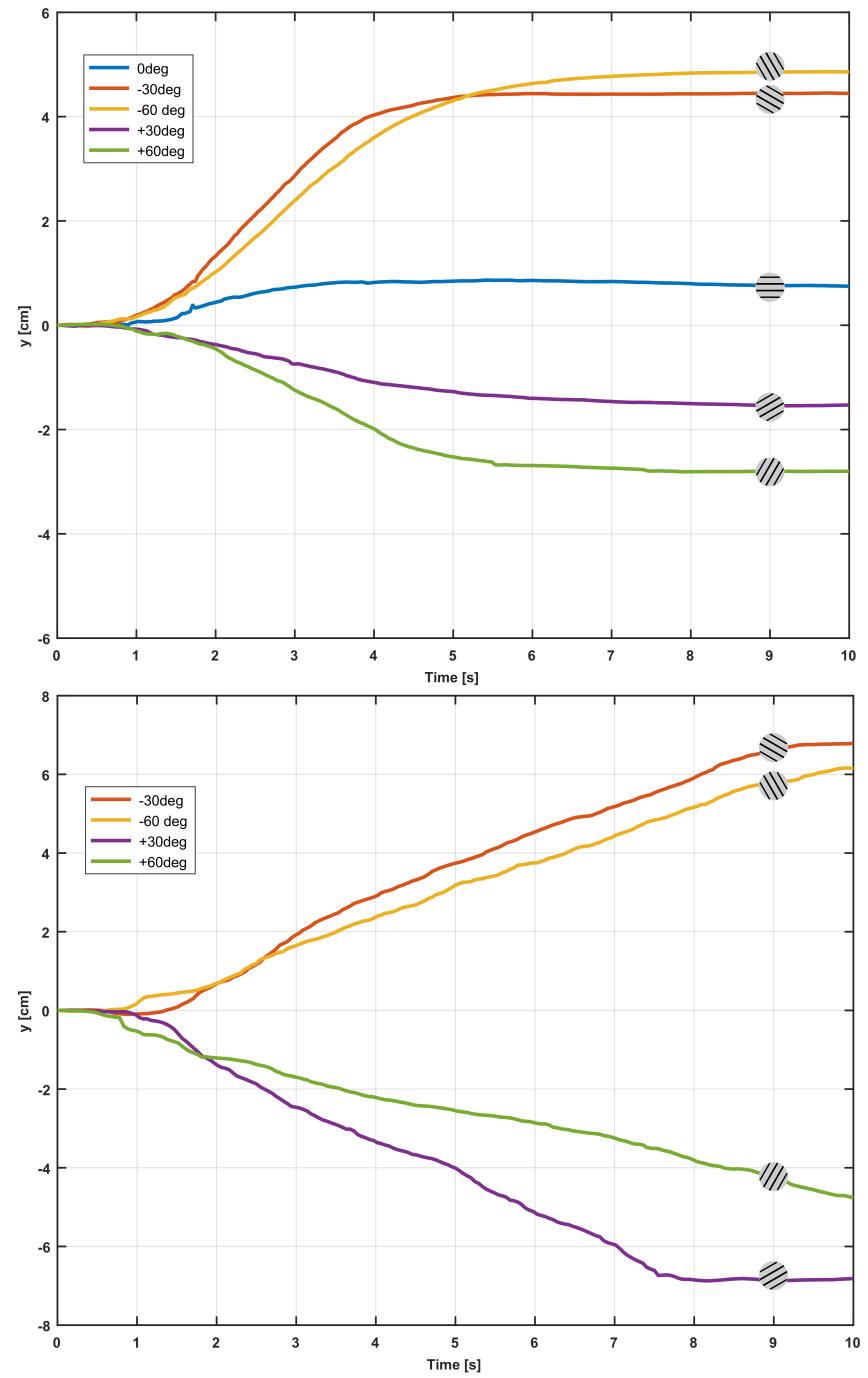

Fig. 5: The position error of the hand trajectory with respect to body mid-line, data from a representative participant. Positive y values are for a leftward deviation from the mid-line, whereas negative values for a rightward deviation. In the top panel, data from the experimental task (the participant was asked to move the hand away from her along a straight path). The deviation from the body mid-line was the largest when the ridges were oriented at $\pm 60 \mathrm{deg}$, in accordance with model predictions. In the bottom panel, the blindfolded participant was instructed to follow the ridges. Following the ridges at \pm 30 deg produced the largest deviation from body mid-line. Ridge orientations are labeled in figure legend and with an oriented plate on top of each line.

and $0.02 \mathrm{~N}$ (peak at $30 \mathrm{deg}$ counterclockwise minus peak at 30 deg clockwise).

\section{Discussions AND Future WORKS}

In this paper, we showed that, when we slide our finger over a ridged surface, cutaneous touch affects the direction of the active movement. According to our hypothesis, and coherently with tactile flow model, tactile feedback produced an illusory sensation of bending towards a direction perpendicular to the ridges and this triggered a correction of the movement in the opposite direction. As shown in [18], the position of the plate with respect to the frontal and the sagittal plane of the participant can also affect the perceived hand orientation and motion. Accordingly, moving on a smooth plane without ridges, participants deviated their hand trajectory of approximately $4 \mathrm{deg}$ from the straight direction.

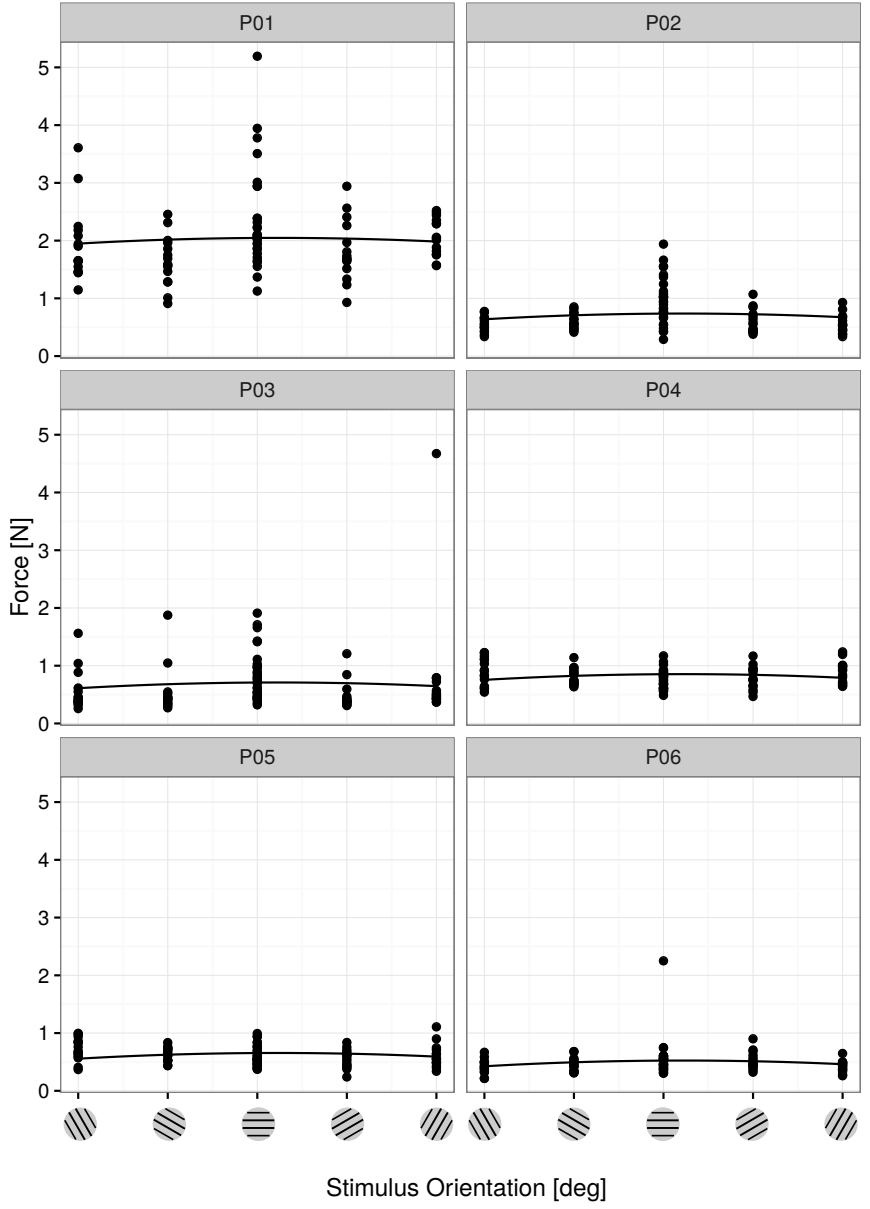

Fig. 6: The force peak as a function of the orientation of the grating in participants P01-P06. Point data for individual trial and LMM prediction.

This extra-cutaneous bias accounted for the asymmetry of the results between the clock- and the counterclockwise rotation of the stimuli (Fig. 4). In the present study, the hand motion was self-paced, producing a random variation in motion speed. In future experiments, the effect of the hand speed to modulate the phenomenon can be also investigated.

Receptors from the muskoloskeletal system and the skin, in addition to the efferent copy, provide information about the static position and movement of our hand and our limbs. Here we assumed that the integrated estimate of the hand displacement $\hat{V}$ was equal to a weighted sum of the estimate from tactile slip and the ones from the other cues.

$$
\hat{V}=\hat{w}_{\text {prop }} \hat{V}_{\text {prop }}+\hat{w}_{\text {tact }} \hat{V}_{\text {tact }}
$$

where $\hat{V}, \hat{V}_{\text {prop }}, \hat{V}_{\text {tact }}$ are the integrated, the proprioceptiveefferent and the tactile slip estimate, respectively. Note that in our experimental paradigm, it was not possible to evaluate separately the individual contribution of the receptors in the muskoloskeletal system, of the skin stretch and of the efferent copy. Therefore, in Equation (5) we labeled the displacement estimate provided by the efferent copy and by all the other proprioceptive cues except for tactile slip as $\hat{V}_{\text {prop }}$. We can provide a preliminary estimate of the relative contribution of tactile slip, as follows. We assumed that the direction of $\hat{V}$ (i.e., the perceived hand direction) was always aligned with 
the sagittal plane, as required by the task. For simplicity, we assumed that $\hat{V}_{\text {prop }}$ was unbiased and therefore aligned with the actual motion trajectory. Finally, we assumed $\hat{V}_{\text {tact }}$ to be orthogonal to the ridge orientation, in accordance with the tactile flow model. We estimated $\hat{w}_{\text {prop }}$ as the length of the projection of the actual motion trajectory on the vector $\hat{V}$. This way, it was possible to estimate the relative contribution of tactile slip, $\hat{V}_{\text {tact }}$, which was approximately equal to 0.13 . The relatively high weight of proprioceptive cues in the perception of hand motion is in accordance with previous studies in the literature [20], [21], [22]. In future studies we aim to develop a theoretical model, e.g. based on the usage of Kalman filtering techniques, accounting for the integration of multisensory information and prior knowledge over time. This way it will be possible to provide an estimate of the dynamic combination of the different cues.

\section{ACKNOWLEDGMENTS}

This work is supported in part by the European Research Council under the Advanced Grant SoftHands "A Theory of Soft Synergies for a New Generation of Artificial Hands" no. ERC-291166, by the EU H2020 project "SOFTPRO: Synergy-based Open-source Foundations and Technologies for Prosthetics and RehabilitatiOn" (no. 688857) and by the EU FP7 project (no. 601165), "WEARable HAPtics for Humans and Robots (WEARHAP)". We thank Priscilla Balestrucci and Colleen P. Ryan for helpful comments and suggestions.

\section{REFERENCES}

[1] R. S. Johansson and G. Westling, "Roles of glabrous skin receptors and sensorimotor memory in automatic control of precision grip when lifting rougher or more slippery objects," Experimental Brain Research, vol. 56(3), pp. 550-564, October 1984.

[2] K. O. Johnson, "The roles and functions of cutaneous mechanoreceptors," Curr. Opin. Neurobiol., vol. 11, no. 4, pp. 455-461, August 2001.

[3] S. J. BensmaIa and M. Hollins, "The vibrations of texture." Somatosensory \& motor research, vol. 20, no. 1, pp. 33-43, jan 2003.

[4] W. Bergmann Tiest and A. Kappers, "Cues for haptic perception of compliance," IEEE Transactions on Haptics, vol. 2, no. 4, pp. 189199 , oct 2009.

[5] M. Bianchi and A. Serio, "Design and characterization of a fabricbased softness display," Haptics, IEEE Transactions on, vol. 8, no. 2, pp. 152-163, April 2015.

[6] A. Moscatelli, M. Bianchi, A. Serio, A. Terekhov, V. Hayward, M. O. Ernst, and A. Bicchi, "The change in fingertip contact area as a novel proprioceptive cue," Current Biology, vol. 26, no. 9, pp. 1159-1163, 2016.

[7] A. V. Terekhov and V. Hayward, "The brain uses extrasomatic information to estimate limb displacement," in Proc. R. Soc. B, vol. 282, no. 1814. The Royal Society, 2015, p. 20151661.

[8] U. Proske and S. C. Gandevia, "The proprioceptive senses: their roles in signaling body shape, body position and movement, and muscle force," Physiological reviews, vol. 92, no. 4, pp. 1651-1697, 2012.

[9] H. C. Bastian, "The 'muscular sense': its nature and cortical localisation," Brain, vol. 10, pp. 1-137, 1888.

[10] B. B. Edin and J. H. Abbs, "Finger movement responses of cutaneous mechanoreceptors in the dorsal skin of the human hand," J Neurophysiology, vol. 65, no. 3, pp. 657-670, 1991.

[11] B. B. Edin and N. Johansson, "Skin strain patterns provide kinaesthetic information to the human central nervous system." The Journal of physiology, vol. 487, no. 1, pp. 243-251, 1995.

[12] D. F. Collins, K. M. Refshauge, G. Todd, and S. C. Gandevia, "Cutaneous receptors contribute to kinaesthesia at the index finger, elbow and knee," J Neurophysiology, vol. 94, pp. 1699-1706, 2005.

[13] N. S. Weerakkody, D. A. Mahns, J. L. Taylor, and G. S. C, "Impairment of human proprioception by high-frequency cutaneous vibration," J Physiology, vol. 581, pp. 971-980, 2007.
[14] C. Blanchard, R. Roll, J. P. Roll, and A. Kavounoudias, "Combined contribution of tactile and proprioceptive feedback to hand movement perception," Brain Research, vol. 1382, pp. 219-229, 2011. [Online]. Available: http://dx.doi.org/10.1016/j.brainres.2011.01.066

[15] A. Moscatelli, A. Naceri, and M. O. Ernst, "Path integration in tactile perception of shapes," Behavioural Brain Research, vol. 274, pp. 355364, nov 2014.

[16] A. Moscatelli, M. Bianchi, A. Serio, A. Bicchi, and M. O. Ernst, "Sensorymotor synergies: Fusion of cutaneous touch and proprioception in the perceived hand kinematics," in Human and Robot Hands. Springer International Publishing, 2016, pp. 87-98.

[17] A. Bicchi, E. P. Scilingo, E. Ricciardi, and P. Pietrini, "Tactile flow explains haptic counterparts of common visual illusions," Brain Res Bull., vol. 75, no. 6, pp. 737-741, 2008.

[18] A. M. L. Kappers and J. J. Koenderink, "Haptic perception of spatial relations," Perception, vol. 28, no. 6, pp. 781-795, 1999.

[19] D. Bates, M. Mächler, B. Bolker, and S. Walker, "Fitting Linear Mixed-Effects Models Using lme4," Journal of Statistical Software, vol. 67, no. 1, pp. 1-51, jun 2015 .

[20] R. J. van Beers, A. C. Sittig, and J. J. Gon, "Integration of proprioceptive and visual position-information: An experimentally supported model." Journal of neurophysiology, vol. 81, no. 3, pp. 1355-64, mar 1999.

[21] M. Tagliabue and J. McIntyre, "When Kinesthesia Becomes Visual: A Theoretical Justification for Executing Motor Tasks in Visual Space," PLoS ONE, vol. 8, no. 7, 2013.

[22] M. Chancel, C. Blanchard, M. Guerraz, A. Montagnini, and A. Kavounoudias, "Optimal visuo-tactile integration for velocity discrimination of self-hand movements." Journal of neurophysiology, p. jn.00883.2015, 2016. 